\title{
Données expérimentales sur le cycle biologique de Petasiger caribbensis n. sp.
}

\author{
(Trematoda : Echinostomatidae)
}

parasite larvaire de Biomphalaria glabrata en Guadeloupe

\author{
par H. NASSI \\ Département de Biologie animale (Directeur : $P^{r}$ C. Combes), \\ Université, avenue de Villeneuve, 66025 Perpignan Cedex (France).
}

\section{Résumé.}

Les stades larvaires (rédie, cercaire, métacercaire) et l'adulte de Petasiger caribbensis, une nouvelle espèce d'Echinostome à 19 épines céphaliques sont décrits. En Guadeloupe, le premier hôte intermédiaire est le Mollusque vecteur de Schistosoma mansoni, Biomphalaria glabrata, lequel est stérilisé par les larves de cet Echinostome. Les métacercaires ont été trouvées dans la nature chez Tilapia mossambica et ont été obtenues expérimentalement chez ce Poisson ainsi que chez Poecilia reticulata, Xiphophorus hellerie et Gambusia affinis. Les adultes se développent expérimentalement chez le canari, mais non chez le caneton ou le poussin. L'hôte définitif naturel est inconnu. Les caractères des stades larvaires sont pris en compte dans la comparaison avec les espèces voisines du genre Petasiger dont le cycle biologique est connu.

\section{Summary.}

Experimental data on the life of Petasiger caribbensis n. sp. (Trematoda : Echinostomatidae) a larval parasite of Biomphalaria glabrata in Guadeloupe.

The larval stages (redia, cercaria, metacercaria) and adult of Petas:ger caribbensis a new Echinostomatid with 19 collar spines are described. In Guadeloupe, the first intermediate host is the snail vector of Schistosoma mansoni, Biomphalaria glabrata, which is sterilized by the larvae of this Echinostome. The metacercariae were recovered in nature from Tilapta mossambica and were obtained experimentally in this fish as well as in Poecilia reticulata, Xiphophorus hellerie and Gambusia affinis. Adults develop experimentally in canaries, but

Accepté le 30 octobre 1979. 
not in ducklings or chicks. The natural definitive host is unknown. The larval characteristics are taken into account in the comparison with related species of the genus Petasiger whose life cycles are known.

\section{Introduction}

Biomphalaria glabrata (Say) est le seul Mollusque vecteur de Schistosoma mansoni en Guadeloupe. Dans cette île, B. glabrata héberge également les stades larvaires de plusieurs Trématodes dont certains déterminent une stérilité totale et définitive de ce Mollusque (Golvan et al., 1975). Par cet impact très marqué sur la fonction reproductrice, ces Trématodes nous sont apparus comme un matériel intéressant pour des recherches orientées vers une lutte biologique contre le vecteur de Schistosoma mansoni. De telles recherches impliquaient la réalisation expérimentale des cycles biologiques de ces différents Trématodes dans le but de déterminer quel était le plus intéressant sur le double plan de la facilité d'entretien du cycle et de l'efficacité. C'est ainsi que Ribeiroia marini guadeloupensis Nassi, 1978 (Cathaemasiidae) fut sélectionné et utilisé avec succès dans un essai de lutte contre une population naturelle de B. glabrata (Nassi et al., 1979). Parmi les cycles élucidés au cours de ce travail de sélection figure celui d'un Echinostome à cercaire macrocerque et dont l'adulte doit être rapporté au genre Petasiger Dietz, 1909. Les comparaisons effectuées avec les autres espèces de ce genre nous ont conduit à considérer l'Echinostome de Guadeloupe comme une nouvelle espèce.

\section{Matériel et méthodes}

Les Biomphalaria glabrata parasités et les Tilapia mossambica hébergeant les métacercaires ont été récoltés dans un étang en voie de comblement de la partie est de l'île (Grande-Terre). Les métacercaires ont été obtenues par infestation de Poissons nés au laboratoire: T. mossambica, Poecilia reticulata, Xiphophorus helleri et Gambusia affinis. Pour ces trois dernières espèces, l'émission quotidienne d'un seul Mollusque permet d'infester plusieurs individus. Les métacercaires, en nombre indéterminé, ont été administrées oralement à des canetons, des poussins et des canaris. Les stades larvaires ont été étudiés sur le vivant. Les adultes fixés au Bouin ont été étudiés après coloration au carmin boracique et montage in toto ; leur anatomie a été précisée par l'examen de coupes sériées de $5 \mu \mathrm{m}$ d'épaisseur colorées à l'Azan de Heindenhain. Toutes les dimensions sont données en micromètres.

\section{Résultats}

Description de l'adulte. (fig. 1).

Echinostomatidae: Echinostomatinae. Corps d'allure générale fusiforme atteignant sa largeur maximum au voisinage du bord antérieur de l'acétabulum et se 
rétrécissant progressivement vers l'arrière. Acétabulum puissant en position approximativement équatoriale. Ventouse orale terminale d'un diamètre égal au tiers de celui de l'acétabulum. Couronne céphalique comprenant toujours 19 épines, dont 8 organisées en 2 groupes ventrolatéraux de 4 épines, et 11 disposées dorsalement sur une seule rangée. Epines tégumentaires squamiformes très denses dans la partie antérieure de la région préacétabulaire. Dorsalement, les épines dépassent rarement le niveau du bord antérieur de l'acétabulum. Ventralement, les épines sont nettement moins denses à partir de l'acétabulum; à partir du testicule antérieur, elles ne sont présentes que dans la région médioventrale et disparaissent complètement en arrière du testicule postérieur. Prépharynx étroit. Bifurcation intestinale située nettement en avant de l'acétabulum ; caecums intestinaux se terminant à mi-distance entre le testicule postérieur et l'extrémité postérieure du corps. Testicules toujours disposés en diagonale et étroitement accolés l'un à l'autre, le testicule antérieur étant en grande partie situé à gauche de l'axe de symétrie du corps. Spermiductes naissant ventralement au pôle supérieur de chaque testicule; le spermiducte chemine vers l'avant du côté gauche pour le testicule antérieur, du côté droit pour le testicule postérieur. Les spermiductes s'abouchent à la base d'une vésicule séminale bipartite qui fait souvent saillie sur le bord postérieur de la poche du cirre. Poche du cirre massive renfermant de nombreuses cellules prostatiques; elle s'appuie en avant sur la bifurcation intestinale et déborde en arrière sur l'acétabulum. Pars prostatica bien développée ; au repos, elle est télescopée sur elle-même et le cirre apparaît mal individualisé. Le débouché du conduit génital mâle dans l'atrium génital se présente sous la forme d'une fente transversale mesurant environ $50 \mu \mathrm{m}$ de long. Le pore génital s'ouvre légèrement à gauche de l'axe de symétrie du corps, au voisinage immédiat, et légèrement en arrière de la bifurcation intestinale. Lorsqu'il s'évagine, l'appareil copulateur mâle entraîne un grand nombre de cellules prostatiques; il se présente alors sous la forme d'un bulbe dont la surface est ornée de microexpansions papilliformes. Ovaire sphérique dorsal situé au voisinage immédiat ou légèrement en arrière de l'acétabulum, toujours à droite de l'axe de symétrie du corps. L'oviducte qui naît ventralement au pôle inférieur de l'ovaire se coude en direction dorsale pour cheminer transversalement vers la gauche, en position dorsale par rapport à l'ootype et parallèlement au grand axe de celui-ci. Le canal de Laurer s'ouvre dorsalement en position médiane et en avant de l'ootype. Avant d'aboutir à l'ootype, l'oviducte reçoit le vitelloducte commun; il y a un réservoir vitellin, dorsal par rapport à l'ootype et toujours situé au niveau du bord antérieur du testicule antérieur, alimenté par deux vitelloductes transverses approximativement horizontaux. Ootype volumineux, avec glandes de Mehlis constituant un organe ovoïde bien délimité situé en grande partie en avant du testicule antérieur et dorsalement par rapport à celui-ci ; son grand axe est transversal et remonte légèrement vers la gauche. C'est du côté gauche et dorsalement que l'oviducte pénètre dans l'ootype. Utérus court remontant dorsalement par rapport à l'acétabulum et du côté gauche. Le métraterme à paroi épaisse forme une poche allongée dont la lumière est ornée de nombreuses expansions cellulaires. Le nombre maximum d'œufs présents dans l'utérus est de 4 . Glandes vitellogènes disposées en 


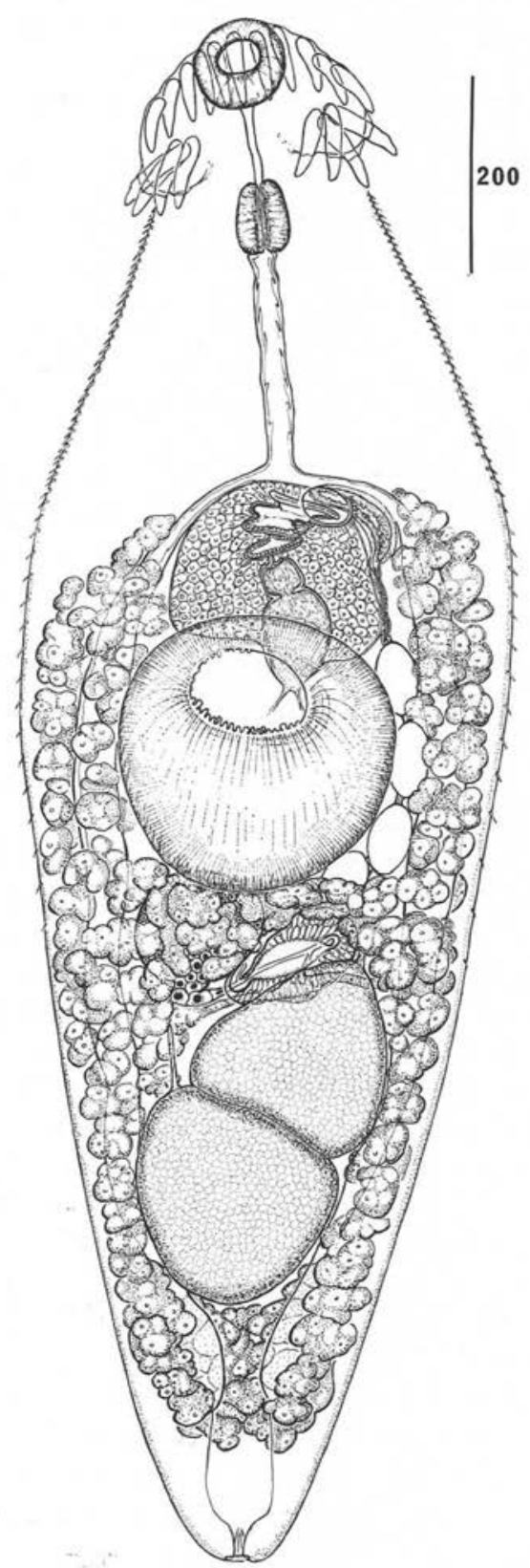

Fig. 1. Adulte de Petasiger carribensis, vue ventrale. Echelle en $\mu \mathrm{m}$. 
deux champs latéraux recouvrant ventralement et dorsalement les caecums. Leur limite antérieure se situe légèrement en arrière de la bifurcation intestinale ; à ce niveau, ils ont tendance à s'étendre en direction médiane et peuvent même confluer dorsalement chez certains individus. Chez tous nos exemplaires, les follicules envahissent l'espace ventral compris entre l'acétabulum et le testicule antérieur, recouvrant partiellement l'ovaire, l'ootype et la partie proximale de l'utérus. Postérieurement, les vitellogènes dépassent légèrement l'extrémité des caecums et les deux champs latéraux fusionnent toujours dorsalement en arrière du testicule postérieur.

Principales dimensions relevées chez 23 spécimens (dans l'ordre longueur sur largeur). Corps : 1250 à 1580 sur 400 à 520 ; longueur région préacétabulaire et postacétabulaire : 446 à 623 et 520 à 734 respectivement; diamètre transversal de la couronne céphalique : 210 à 270 ; longueur épines céphaliques dorsales et ventrolatérales : 64 à 79 et 70 à 95 respectivement; ventouse orale : 70 à 90 sur 70 à 106 ; acétabulum : 235 à 286 sur 235 à 264 ; pharynx : 57 à 78 sur 41 à 58 ; testicule antérieur: 140 à 220 sur 160 à 225 ; testicule postérieur: 144 à 214 sur 180 à 230 ; ovaire : 95 à 135 sur 100 à 136 ; œufs (non déformés à la fixation) : 70 à 84 sur 48 à 55 .

Hôte naturel: inconnu. L'hôte possible, sinon probable, Podilymbus policeps antillarum Bangs, le seul Grèbe signalé par Pinchon (1963) en Guadeloupe ne figure pas parmi les Oiseaux examinés par Euzéby et Graber (1975). La possibilité d'un passage chez le petit Héron vert Butorides virescens maculatus (Boddaert) est sans doute à exclure; en effet, l'exemplaire que nous avons capturé dans la station où les Mollusques et les Poissons étaient fortement infestés s'est révélé négatif, comme de nombreux autres spécimens examinés en Guadeloupe.

Hôte expérimental : canari (essais d'infestation négatifs avec le canard domestique et le poussin). Les dissections de canaris pratiquées entre le $8^{\mathrm{e}}$ et le $11^{\mathrm{e}}$ jour après l'infestation ne nous ont livré que des individus immatures. Cet hôte expérimental n'est pas très favorable car, même au bout de 16 jours, certains des spécimens récoltés n'étaient pas ovigères. Les adultes sont localisés dans la partie antérieure du duodénum.

\section{Description des stades larvaires. (fig. 2).}

Au niveau du premier hôte intermédiaire, nous ne disposons que d'observations réalisées sur des Mollusques infestés naturellement.

\section{RÉDIES :}

Nos observations concernent uniquement les rédies productrices de cercaires, donc certainement des rédies de deuxième et peut-être même de troisième génération. Ces rédies sont de couleur jaune orangé pâle. Elles sont présentes en quantité importante dans la région de l'ovotestis, mais colonisent également les autres régions du Mollusque. Elles sont plutôt longues et très actives. Elles possèdent un collier antérieur constitué de quatre lobes et une paire d'appendices locomoteurs postérieurs. 

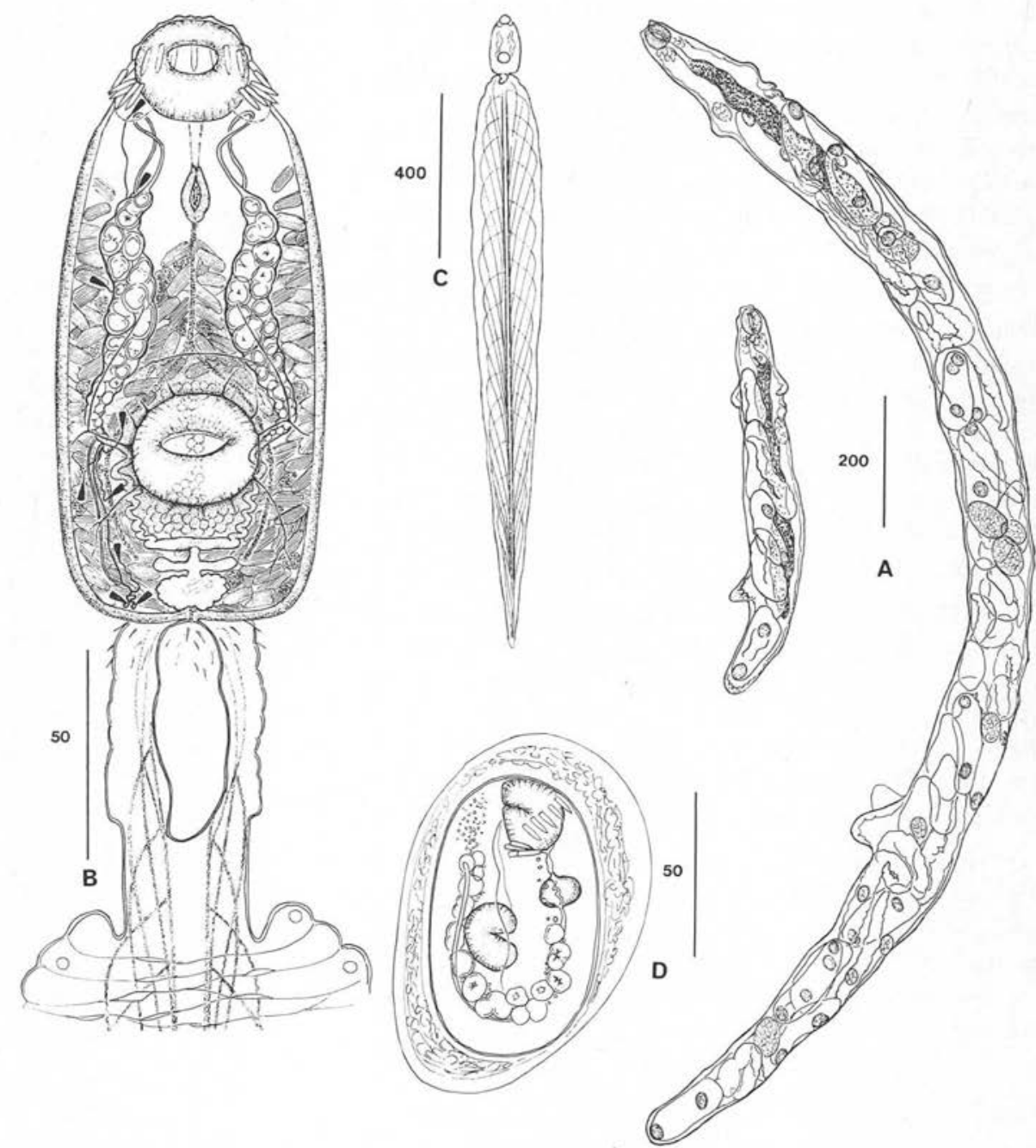

Fig. 2. A. Rédies productrices de cercaires. Noter chez le grand spécimen la brièveté du caecum digestif.

B. Corps cercarien en vue ventrale et pédoncule caudal.

C. Schéma de la cercaire in toto montrant l'agencement des fibres musculaires caudales circulo-diagonales.

D. Métacercaire enkystée.

Echelles en $\mu \mathrm{m}$. 
Le pore de naissance s'ouvre en arrière du lobe dorsal du collier antérieur. Chez les petits spécimens, le cæcum digestif dépasse les appendices postérieurs; chez les plus grands, sa longueur est comprise entre le quart et le tiers de la longueur du corps. Principales dimensions d'un grand spécimen en extension: longueur 2300 ; largeur maximum : 140 ; pharynx: 42 sur 36 ; extrémité antérieure - pore de naissance : environ 200; appendice locomoteur - extrémité postérieure : environ 600.

\section{Cercaire :}

La cercaire est incolore. Le corps est subsphérique lorsqu'il est contracté et pratiquement rectangulaire en extension moyenne. Les épines de la couronne céphalique sont disposées comme chez l'adulte; nous en avons compté 19 chez tous les exemplaires étudiés à l'exception d'un seul qui en possédait 20 . La ventouse orale et la ventouse ventrale sont sub-égales, le tégument est épais et la fine spinulation signalée chez toutes les cercaires du même type n'a pu être observée. Ventralement, en arrière du pharynx, le tégument est granuleux; il s'épaissit considérablement dans la partie moyenne du corps et forme à la périphérie de l'acétabulum un bourrelet plissé radialement. Ce bourrelet se déforme constamment et peut constituer deux lèvres transversales qui viennent recouvrir partiellement la partie antérieure et la partie postérieure de l'acétabulum. Le prépharynx est relativement long; la lumière du pharynx est remplie d'un pigment jaune vif. Le reste du tube digestif est peu différencié, l'œsophage et les cæecums n'étant représentés que par une étroite bande de matériel granuleux. Les ébauches cæcales atteignent la chambre antérieure de la vessie. Il y a 18 protonéphridies organisées suivant la formule : $2[(3)+(3)+(3)]$. L'une des protonéphridies du groupe para-acétabulaire n'a pu être observée chez un grand nombre de cercaires probablement à cause de l'abondance des cellules kystogènes. Le tube excréteur naît au niveau du pharynx de la fusion des canalicules des 3 protonéphridies antérieures; il reçoit successivement les canalicules des flammes para-acétabulaires et para-vésicales, atteignant l'extrémité postérieure du corps. Il remonte jusqu'à la ventouse orale, sa lumière étant alors pourvue de bouquets de cils pulsateurs. Il se courbe ensuite en direction ventrale et redescend jusqu'à la vessie. Entre le pharynx et l'équateur acétabulaire, le tube collecteur primaire est très dilaté et renferme une trentaine de concrétions réfringentes. Dans la région acétabulaire, ces concrétions sont constituées d'un granule simple. Au-dessus de l'acétabulum, leur taille est plus importante et elles sont constituées pour la plupart de l'association de deux et parfois trois granules enfermés à l'intérieur d'une enveloppe réfringente commune. Les tubes collecteurs droit et gauche confluent en un court canal commun aboutissant à la chambre antérieure de la vessie qui est constituée de deux lobes. La chambre postérieure de la vessie, de forme simple, semble être en relation avec une vésicule de grande taille située à la base de la queue. L'ébauche génitale est représentée par deux massifs cellulaires, l'un anté-acétabulaire, l'autre plus volumineux post-acétabulaire, reliés entre eux par une étroite file de cellules. Les cellules kystogènes sont nombreuses mais font défaut dans la région antérieure du corps et dorsalement dans la région de l'acétabulum. Ces cellules renferment de nombreux bâton- 
nets organisés en faisceau, ce qui leur confère une forme grossièrement parallélépipé dique. A son insertion sur le corps, la queue a un diamètre réduit; elle forme un pédoncule qui est le plus souvent contracté et invaginé dans l'axe caudal. A sa base, le pédoncule caudal porte trois à quatre rangées irrégulières d'épines de longueur variable (2 à $6 \mu \mathrm{m})$. Le tégument caudal présente une annélation peu marquée. La structure caudale la plus visible est constituée de fibres musculaires à disposition circulo-diagonales. Chacune de ces fibres naît apparemment en position para-axiale et prend immédiatement une direction postéro-latérale; puis elle revient progressivement en position para-axiale, rejoignant ainsi les fibres nées plus haut, et chemine longitudinalement sur une courte distance avant de s'interrompre. Durant leur trajet, il semble que ces fibres parcourent une demi-circonférence. Il y aurait ainsi deux ensembles de fibres droit et gauche symétriques par rapport au plan sagittal; cette disposition pourrait être en relation avec le léger aplatissement dorso-ventral de la queue. Outre leur rôle locomoteur, ces fibres pourraient également avoir comme fonction de maintenir la rigidité de la queue au repos.

Principales dimensions (dans l'ordre longueur sur largeur) : corps : 100 sur 110 en contraction, 200 sur 60 en extension; queue : 1100 à 1400 sur 150 à 190 ; ventouse orale : 26 à 30 sur 28 à 34 ; acétabulum : 26 à 32 sur 30 à 36 ; longueur des épines céphaliques : épines dorsales : 7 à 8 , épines ventro-latérales : 7 à 9 ; longueur du pharynx : 12 à 15.

\section{MÉTACERCAIRE :}

Le deuxième hôte intermédiaire est un Poisson qui s'infeste soit en ingérant passivement les cercaires entraînées par les courants d'eau respiratoires, soit en les consommant activement. Les métacercaires se localisent essentiellement dans la paroi du pharynx et de la région antérieure de l'œsophage. Les corps cercariens traversent la muqueuse avant de s'enkyster. La paroi du kyste est au départ uniquement d'origine parasitaire et ne mesure que 3 à $4 \mu \mathrm{m}$ d'épaisseur. Par la suite, elle va se doubler d'une enveloppe réactionnelle fibreuse mise en place par l'hôte et dont l'épaisseur peut atteindre plusieurs dizaines de microns. Lorsque l'infestation est massive, plusieurs métacercaires peuvent être incluses dans une même enveloppe réactionnelle. Ces kystes peuvent être suffisamment volumineux pour déformer la face externe de l'œsophage. Débarrassés de l'enveloppe réactionnelle, les kystes mesurent en moyenne $85 \mu \mathrm{m}$ de long sur $53 \mu \mathrm{m}$ de large. La métacercaire est repliée sur sa face ventrale, la ventouse orale venant au contact de l'extrémité postérieure du corps. Il n'y a pas de transformations importantes au cours de la maturation. Ainsi, il est à noter que le pigment pharyngien subsiste. Les modifications les plus évidentes concernent le tégument dont la spinulation apparaît et le tube digestif dont le diamètre augmente et dont la lumière devient visible à la partie supérieure de l'œsophage à partir du $8^{\mathrm{e}}$ jour. Il semble que le délai de maturation des métacercaires soit assez long ; l'infestation du canari n'a été possible en effet qu'avec des métacercaires âgées d'au moins deux semaines. 
Dans la nature, le rôle de leurre joué par la queue privilégie sans aucun doute le mode d'infestation par consommation du Poisson deuxième hôte intermédiaire, ce mode d'infestation étant en outre favorisé par les modalités de l'émission cercarienne. En effet, Théron (1975) a montré que pour une LD 12 : 12, l'émission débute en fin de période obscure et atteint son maximum une heure avant l'éclairement. Plus de $65 \%$ des cercaires sont ainsi émises au cours des trois dernières heures d'obscurité, ce pourcentage atteignant près de $80 \%$ après une heure d'éclairement. Dans la nature, cette émission massive à la fin de la nuit et en début de matinée assure une concentration importante de cercaires dans le milieu dès la reprise de l'activité trophique des Poissons. Le phototropisme positif très marqué des cercaires amène celles-ci dans les couches d'eau les plus superficielles, ce qui favorise probablement l'infestation des Poissons qui mangent préférentiellement en surface, comme les Poecilidés. L'infestation expérimentale de Poecilia reticulata (Guppy), l'un des hôtes naturels probables en Guadeloupe, ou d'autres Poecilidés comme Xiphophorus helleri et Gambusia affinis, se traduit souvent par une hyperparasitose due à leur voracité vis-à-vis de ces cercaires. Le rendement de l'infestation de Tilapia mossambica, chez lequel des métacercaires ont été trouvées dans la nature, est plus faible.

\section{Discussion}

Dans le genre Petasiger Dietz, 1909, le nombre d'espèces dont le cycle biologique est connu, partiellement ou en totalité, est réduit. Pour le Nouveau Monde, il s'agit de $P$. nitidus Linton, 1928 et de $P$. chandleri Abdel Malek, 1952 en Amérique du Nord, et de $P$. novemdecim Lutz, 1928 en Amérique du Sud. Pour le reste du Monde, il s'agit de $P$. neocomense Fuhrmann, 1927 dans la région aralo-caspienne et de $P$. australis Johnston et Angel, 1941 en Australie.

Si l'on prend en considération à la fois les caractères des stades larvaires et ceux des adultes, des différences apparaissent entre le Trématode guadeloupéen et chacune de ces cinq espèces.

Linton (1928) a décrit $P$. nitidus d'après des spécimens trouvés chez Podiceps auritus (L.). Le cycle de cette espèce a été élucidé par Beaver (1939) ; c'est à la description des adultes rapportée par Beaver que nous nous référerons, car ces adultes ont été obtenus expérimentalement chez le canari, comme pour le Trématode de Guadeloupe. Chez cet hôte expérimental, le délai de maturation des adultes de $P$. nitidus est de neuf jours, délai nettement plus court que celui que nous avons observé. Sur le plan morphologique, la différence la plus évidente réside dans l'absence chez $P$. nitidus de vitellogènes dans l'espace ventral compris entre l'acétabulum et les organes génitaux. Il semble en outre que l'ootype et le réservoir vitellin occupent une position plus postérieure chez $P$. nitidus. En effet, Beaver décrit et représente l'ootype sous le testicule antérieur et le réservoir vitellin au niveau du bord postérieur de ce testicule ; chez les spécimens guadeloupéens l'ootype est en grande partie situé en avant du testicule 
antérieur et le réservoir vitellin plutôt au niveau du bord antérieur de ce testicule. Enfin, les dimensions moyennes des œufs de P. nitidus $(90 \times 60)$ sont supérieures aux dimensions maximales que nous avons relevées. Il existe également des différences au niveau des stades larvaires qui évoluent pour $P$. nitidus chez Helisoma antrosum percarinatum (Walker) et $H$. campanulatum smithii (Baker). Chez les rédies de $P$. nitidus, le cæcum est du type long et atteint les appendices postérieurs. Chez la cercaire, le pharynx est nettement plus petit (6 sur 8 ) et renferme un pigment brun foncé; Beaver décrit 3 paires de cellules para-œsophagiennes que nous n'avons pas observées, mais ne signale ni le bourrelet péri-acétabulaire ni les épines du pédoncule caudal.

Le cycle de $P$. chandleri a été élucidé par Abdel Malek $(1952,1953)$. Cet auteur a décrit l'adulte à partir de spécimens récoltés dans la nature chez Podilymbus podiceps L. et n'a pu obtenir que des immatures chez le canari, même après un délai de 14 jours. Au stade adulte, $P$. chandleri se distingue du parasite de Guadeloupe par trois caractères: les cæcums ne dépassent pas le testicule antérieur, il n’y a pas de vitellogènes ventraux entre l'acétabulum et les organes génitaux et le nombre d'épines céphaliques est variable (19 à 21). A ces différences morphologiques qui suffisent d'après nous pour distinguer ces deux espèces viennent s'ajouter celles relevées au niveau des stades larvaires qui évoluent pour $P$. chandleri chez Helisoma corpulentum Say. Le cæcum des rédies est ici encore du type long puisqu'il s'étend au-delà des appendices postérieurs. Chez la cercaire, la queue est nettement plus courte (860), la couronne adorale compte 21 épines, le pigment pharyngien est jaune orangé, les ébauches cæcales, plus larges que l'œsophage, ne dépassent pas le milieu de l'acétabulum, et la vessie est indivise; ici encore ni le bourrelet périacétabulaire, ni les épines du pédoncule caudal ne sont signalées.

Des deux espèces nord-américaines, c'est de $P$. chandleri que le Trématode de Guadeloupe s'éloigne le plus sur le plan morphologique.

Le parasite guadeloupéen se distingue également de $P$. novemdecim dont le cycle a été élucidé par Nasir et al. (1972), bien que cette espèce ait comme premier hôte intermédiaire B. glabrata (sous-espèce olivacea). La description de l'adulte par Nasir et al. porte sur des spécimens récoltés dans la nature chez Podiceps dominicus speciosus (Lynch Arribalzaga), ces auteurs n'ayant pu obtenir d'adultes ovigères chez les jeunes de ce Grèbe infestés en laboratoire. En nous référant à la fois à leur description et à leur dessin, nous relevons les différences suivantes : le nombre d'épines céphaliques est variable ((19 à 21 , rarement 23$)$, les épines céphaliques ventrolatérales sont plus longues (jusqu'à 130), les champs vitellins latéraux débutent plus en arrière et ne confluent pas en arrière du testicule postérieur, les œufs sont plus petits (60 à 72 sur 30 à 45). L'ovaire dont la position par rapport à l'axe de symétrie n'est pas précisée dans la description est figuré à gauche sur le dessin. Des différences s'observent également au niveau des stades larvaires. Les rédies de $P$. novemdecim sont plus petites (longueur maximum 846). Chez la cercaire il y a 19 à 21 épines céphaliques (parfois 23), l'œsophage renferme un pigment brun jaunâtre, la queue est foliacée et nettement plus courte (longueur maximum 451) et surtout l'excréteur compte 24 pro- 
tonéphridies; comme pour les deux espèces précédentes, ni le bourrelet péri-acétabulaire, ni les épines du pédoncule caudal ne sont décrits.

Pour le Nouveau Monde, en dehors de ces trois espèces, il convient de mentionner l'existence d'une cercaire macrocerque à 19 épines céphaliques émise à PortoRico par B. glabrata. Cette cercaire a été signalée successivement par Marin (1928) et Faust et Hoffman (1934). La description de Marin qui est très succincte précise seulement que la couronne céphalique possède 19 épines et que la vessie est formée d'une chambre antérieure et d'une chambre postérieure. Cet auteur ne signale pas de pigment pharyngien et donne des dimensions nettement inférieures aux nôtres (corps : 90 de long sur 68 de large ; queue : 510 de long). Les rédies sont également plus petites (825 de long sur 120 de large). Faust et Hoffman qui ont décrit cette cercaire sous le nom de Cercaria paucispina estiment que les dimensions données par Marin pour le corps sont erronées; d'après eux le corps mesure $200 \mu \mathrm{m}$ de long sur 100 de large. Ces valeurs sont proches des nôtres ainsi que celle donnée pour le diamètre du pharynx (13). Par contre, la queue semble plus courte (4 à 5 fois la longueur du corps) et les ventouses plus grandes (ventouse orale : 30 de long sur 44 de large ; acétabulum : 35 sur 50 ). En outre, le pigment pharyngien n'est pas signalé et l'œsophage se divise presque immédiatement après le pharynx; enfin, comme chez la cercaire de $P$. chandleri, les cæcums ne dépassent pas la partie antérieure de l'acétabulum et la vessie ne comprend qu'une seule chambre. Faust et Hoffman sont les seuls parmi les auteurs cités à signaler la propriété que possède l'acétabulum de s'enfoncer dans les tissus ventraux ou d'en faire saillie. Il est à noter qu'en ce qui concerne la structure de la vessie, Faust et Hauffman sont en contradiction avec Marin qui a décrit et représenté une vessie comprenant deux chambres successives. Compte tenu des différences existant avec la description de Faust et Hoffman et de l'imprécision de celle de Marin, il n'est pas possible d'affirmer l'identité de la cercaire de Guadeloupe avec celle de Porto-Rico.

Le cycle de $P$. neocomense a été élucidé dans la région du delta de la Volga. Le développement des œufs a été étudié par Ginetsinskaia (1952) (in Karmanova, 1971) qui a pu infester expérimentalement Planorbis planorbis L. Dans la nature, les stades larvaires ont été retrouvés chez Gyraulus acronicus (Férussac) par Karmanova (1971) qui a obtenu le développement expérimental des adultes chez de jeunes Grèbes. Nous ne pouvons faire de comparaisons avec ces adultes car Karmanova ne les décrit pas. En conséquence, nous ne pouvons que nous référer à la description originale établie par Fuhrmann (1927) à partir des spécimens trouvés chez Podiceps cristatus (L.) en Suisse. Des différences apparaissent alors qui concernent les dimensions de plusieurs organes. Chez un individu comparable par sa longueur $(1560)$ au plus grand de nos spécimens la couronne céphalique est plus large (320), le pharynx plus volumineux $(90 \times 60)$, le diamètre de l'acétabulum plus important $(340)$, l'ovaire plus petit $(70)$ ainsi que les testicules $(200 \times 50$ et $148 \times 60)$. Trois différences peuvent encore être notées : le cirre est tubulaire, l'ensemble ovaire-testicules est entièrement contenu dans la moitié antérieure de la région post-acétabulaire et les vitellogènes ne confluent pas ventralement entre l'acétabulum et les testicules. 
A côté de la description originale de $P$. neocomense nous devons citer celle qu'Oshmarin (1950) a donné de cette espèce à partir de spécimens également récoltés chez $P$. cristatus en Extrême-Orient soviétique; cet auteur est en effet le seul à avoir décrit chez cette espèce un cirre bulbeux. Nous trouvons ici encore un certain no:nbre de différences. Ainsi, chez l'individu dont Oshmarin donne les principales dimensions, individu comparable par sa longueur (1300) à celle de nos plus petits spécimens, la ventouse orale est plus grande $(105 \times 87)$ ainsi que l'acétabulum (diamètre $=310)$ et le pharynx $(82 \times 62)$. Les œufs sont également plus grands $(96 \times 54)$. Les champs vitellins latéraux qui débutent un peu en avant du bord antérieur de l'acétabulum s'étendent jusqu'à l'extrémité postérieure du corps sans s'unir nulle part l'un à l'autre. Enfin, les testicules occupent une position plus postérieure puisqu'ils sont contenus dans le dernier quart du corps. Nous devons ajouter que l'identité entre le $P$. neocomense de Fuhrmann et celui d'Oshmarin nous semble discutable vu la structure différente du cirre.

Par son organisation générale, la cercaire de $P$. neocomense, telle qu'elle est décrite par Karmanova, est proche de celle de Guadeloupe ; mais par ses dimensions elle en diffère suffisamment pour que l'on puisse considérer les deux espèces comme distinctes (corps : 311 sur 148; diamètre de la ventouse orale et de l'acétabulum : 61, diamètre du pharynx : 23).

L'adulte de P. australis a été décrit en Australie par Johnston et Angel (1941) d'après des spécimens récoltés chez Podiceps ruficollis novaehollandiae Stephens et $P$. poliocephalus Jardine et Selby. Sans en apporter de preuves expérimentales, ces auteurs ont admis que ces adultes correspondaient à Cercaria gigantura Johnston et Angel, 1941 émise par Amerianna pyramidata (Sowerby). La longueur de l'adulte qui ne dépasse pas $700 \mu . \mathrm{m}$ chez l'hôte naturel est de loin inférieure à celle que nous avons nous mêmes relevée et ceci chez un hôte expérimental que l'on peut soupçonner d'être moins favorable au développement. Il n'est pas possible de prendre en considération les dimensions des différents organes de $P$. australis qui sont évidemment plus petits. Cependant, trois rapports dimensionnels sont selon nous significativement différents : rapport longueurs épines ventro-latérales/épines dorsales voisin de 2 ; rapport ventousaire égal à 3,7 ; rapport longueur région post-acétabulaire/largeur du corps égal à 0,75 sur le dessin de Johnston et Angel (1,2 à 1,4 dans notre cas). La valeur de ce dernier rapport traduit la briéveté relative de la région post-acétabulaire, laquelle confère au corps de $P$. australis une forme très différente de celle du parasite guadeloupéen, cette différence étant encore accentuée par l'étranglement de la région préacétabulaire entre le pharynx et la bifurcation intestinale. Quant à la cercaire attribuée à $P$. australis elle est indiscutablement différente de la nôtre car elle possède entre 30 et 36 protonéphridies.

L'ensemble des différences relevées tant au niveau des stades larvaires qu'au niveau des adultes nous conduit à considérer le parasite de Guadeloupe comme une espèce distincte des cinq espèces citées.

L'adulte de Guadeloupe se caractérise entre autre par la constance du nombre d'épines céphaliques. Ce caractère est donc bien fixé chez ce parasite et constitue 
un bon critère de comparaison avec les autres espèces du genre Petasiger dont seul l'adulte est connu. Parmi celles-ci figurent des espèces à testicules en tandem et d'autres à testicules disposés en diagonale ou plus ou moins symétriquement, ces derniers étant placés par Baschkirova (1941) dans le sous-genre Neopetasiger. Bien que nous ralliant à Yamaguti (1958) qui ne reconnaît pas ce sous-genre, nous n'en considérons pas moins que la disposition des testicules est à prendre en compte pour comparer les différentes espèces, contrairement à l'opinion exprimée par Nasir et al. (1972). Chez les adultes de Guadeloupe, la disposition des testicules est encore un caractère constant; ils ne sont jamais disposés en tandem mais toujours en diagonale. Donc parmi les espèces du genre Petasiger connues seulement par l'adulte, le parasite de Guadeloupe ne peut être comparé qu'à celles qui possèdent à la fois 19 épines et des testicules disposés en tandem. Ces espèces sont $P$. grandivesicularis Ishii, 1935, $P$. longicirratus Ku, 1938, P. skrjabini (Baschkirova, 1941), P. floridus Premvati, 1968 et P. pseudoneocomense (Bravo Hollis, 1969).

Nous éliminons d'emblée $P$. longicirratus et $P$. skrjabini qui possèdent un cirre tubulaire et qui parasitent des Anatidés.

Ishii (1935) ne précise pas la structure du cirre de $P$. grandivesicularis qui parasite Podiceps ruficollis japonicus (Hartert) au Japon. Mais cette espèce présente de nombreuses différences avec les spécimens de Guadeloupe : longueur maximum du corps plus faible (636) ; différence de longueur entre épines ventrolatérales et épines dorsales plus grande (respectivement 56 à 61 et 19 à 28) ; rapport ventousaire plus grand (voisin de 4); œsophage plus court; testicules situés dans le quart postérieur du corps ; pore génital médian à mi-distance entre l'acétabulum et la bifurcation intestinale ; champs vitellins confluant au niveau de la bifurcation intestinale.

Premvati (1968) ne précise pas non plus la forme du cirre de $P$. floridus qui parasite Podilymbus podiceps en Floride. Cet auteur indique cependant que la poche du cirre est entièrement pré-acétabulaire, que la pars prostatica est très longue et que le pore génital est situé très loin en arrière de la bifurcation intestinale, trois caractères que nous ne retrouvons pas chez les adultes de Guadeloupe. En outre, chez $P$. floridus, l'acétabulum est plus grand ( 370 à 400 pour une longueur de corps comprise entre 1580 et 2080 ), l'utérus est dextre et les œufs sont non seulement de forme différente mais encore plus petits (60 à 70 sur 40 à 45); enfin, les champs vitellins ne confluent pas entre l'acétabulum et les glandes génitales mais, par contre, envahissent tout l'espace post-testiculaire, y compris l'espace ventral (non précisé dans le texte mais représenté sur le dessin de Premvati).

P. pseudoneocomense parasite le grand Grèbe américain, Aechmophorus occidentalis (Lawrence) au Mexique (Bravo-Hollis, 1969). Comme chez le Petasiger de Guadeloupe, le cirre s'évagine en forme de bulbe. Mais des différences existent qui distinguent les deux parasites. Par rapport à nos spécimens, l'acétabulum est plus grand (287 à 408 sur 302 à 408 pour une longueur de corps comprise entre 830 et 1767 ) et l'ovaire est ovoïde et plus petit ( 82 à 111 sur 61 à 82 ). De plus, les testicules sont toujours disposés symétriquement en " $\mathrm{V}$ » et le réservoir vitellin est ventral. Enfin, 
les champs vitellins qui ne confluent pas entre l'acétabulum et l'ensemble ovairetesticules envahissent tout l'espace post-testiculaire cæcal, intercæcal et extracæcal.

Parmi les espèces à 19 épines, il convient encore de mentionner $P$. pungens (Von Linstow, 1894) et $P$. megacanthum (Kotlan, 1922). Car bien que ces deux espèces aient été initialement décrites avec des testicules en tandem, Prudhoe (1945) a signalé que cette disposition n'était pas constante, et que les testicules pouvaient également être disposés obliquement ou même symétriquement. Les spécimens de Guadeloupe ne peuvent pas être rapportés à $P$. megacanthum, car cette espèce a été décrite avec un cirre tubulaire tant par Kotlan (1922) que par Prudhoe. Pour ce qui est de $P$. pungens, nous nous référerons à la description la plus récente, celle de Prudhoe, qui porte sur des spécimens parasitant Podiceps ruficollis Pallas en Angleterre. Cette espèce qui possède un cirre bulbeux présente avec le parasite de Guadeloupe un nombre suffisant de différences pour distinguer les deux espèces : acétabulum plus important (350 pour une longueur de 1600 d'après la figure de Prudhoe contre 280 pour une longueur de 1580 dans notre cas) ; pharynx plus volumineux (75 à 100 sur 70 à 98); œsophage plus court ; champs vitellins débutant plus en arrière et ne confluant nulle part; œufs plus gros (84 à 96 sur 56 à 61).

Nous considérons donc que le Trématode de Guadeloupe est distinct de toutes les espèces citées. Par ce fait même, nous sommes en désaccord avec Nasir et al. (1972). Ces auteurs considèrent en effet qu'une grande partie des différences d'après lesquelles il nous semble possible de distinguer les adultes ne procèdent que de la variation intraspécifique. Ils sont ainsi amenés à mettre en synonymie avec $P$. pungens (Von Linstow, 1894) toutes les espèces que nous avons mentionnées, à l'exception de $P$. novemdecim dont ils ont réalisé le cycle, et de $P$. pseudoneocomense, le travail de Bravo-Hollis (1969) n'étant pas pris en compte par ces auteurs. Nasir et al. citent à l'appui de leurs conclusions le travail de Prudhoe (1945). Or, si effectivement Prudhoe valide $P$. pungens (Von Linstow, 1894), il n'en considère pas moins cette espèce comme distincte de $P$. megacanthum (Kotlan, 1922); et, parmi les espèces que nous avons citées seules $P$. nitidus Linton, 1928 et $P$. neocomense Fuhrmann, 1927 sont considérées par Prudhoe comme synonymes de $P$. megacanthum (Kotlan, 1922), bien que $P$. nitidus ait un acétabulum nettement plus petit que les deux espèces précédentes.

Au cours de ce travail, nous avons été amenés à mettre l'accent sur l'existence dans le genre Petasiger de deux types d'appareil copulateur, un type tubulaire et un type bulbeux. La prise en compte de ce caractère s'est avérée intéressante pour distinguer les unes des autres certaines des formes à 19 épines que nous avons mentionnées. Pour le moment cependant, il n'est pas possible de l'introduire dans la clé dichotomique du genre Petasiger car la structure du cirre n'est pas connue chez toutes les espèces.

Les différences relevées entre notre matériel et les diverses espèces citées nous amènent à considérer le parasite de Guadeloupe comme une nouvelle espèce pour laquelle nous proposons le nom de Petasiger caribbensis. 


\section{REMERCIEMENTS}

Ce travail a reçu un support financier de la D.G.R.S.T. et de l'I.N.S.E.R.M., et a été réalisé dans le cadre de l'Action Concertée Bilharziose (Dir.: Y.-I. Golvan). La partie terrain a été réalisée à la station de Zoologie de l'I.N.R.A., à Petit-Bourg, Guadeloupe.

\section{Bibliographie}

Abdel Malek E. T. (1952) : Cercaria chandleri, a new echinostome species from the snail Helisoma corpulentum in lake Itasca, Minnesota. Trans. amer. micr. Soc., 71, 277-281.

Abdel Malek E. T. (1953) : Life history of Petasiger chandleri (Trematoda : Echinostomatidae) from the pied-billed grebe, Podilymbus podiceps podiceps with some comments on other species of Petasiger. J. Parasitol., 39, 152-158.

Bashkirova E.I. (1941): In Trematodes of Animals and Man, Echinostomatidae, Skrjabin and Bashkirova, 1956. Akad. Nauk. S.S.S.R., Moskova-Leningrad, 12, 51-930.

Beaver P.C. (1939): The morphology and life history of Petasiger nitidus Linton (Trematoda: Echinostomatidae). J. Parasitol., 25, 269-276.

Bravo Hollis M. (1969): Petasiger (Neopetasiger) pseudoneocomense sp. nov. (Echinostomatidae, Poche, 1926) del intestino de Aechmophorus occidentalis (Lawrence) de Bahia de Todos Santos, Baja California. Ann. Inst. Biol. Mex., ser. Zool., 40, 15-20.

Euzeby J., Graber M. (1975) : Deuxième enquête parasitologique en Guadeloupe. Note I : Les Trématodes des Oiseaux aquatiques. Bull. Soc. Sci. vét. Méd. comp., (Lyon), 77, 317-320.

Faust E. C., Hoffman W. A. (1934) : Studies on schistosomiasis mansoni in Puerto Rico. III. Biological studies. I. The extra-mammalian phases of the life cycle. Puerto Rico J. Publ. Health. Trop. Med., 10, 1-47.

Fuhrmann O. (1927): Petasiger neocomense nov. sp., une nouvelle espèce d'Echinostomatidae. Bull. sioc. Neuchatel Sci. Nat., N.S., 1, 3-6.

Golvan Y.-J., Combes C., Nassi H. (1975): Castration du Mollusque Biomphalaria glabrata par les larves de divers Trématodes guadeloupéens. C.R. Acad. Sci. Paris, sér. D, 280, 1607-1610.

Ishii N. (1935) : Studies on bird trematodes. III. Bird trematodes in Japan (continued). IV. Seven new bid trematodes. Jap. Experiment. Med., 13, 275-284.

Johnston T.H., Angel M. L. (1941) : Life history of the trematode Petasiger australis n. sp. Trans. Soc. Austral., 65, 285-291.

Karmanova E.M. (1971): The life cycle of Petasiger neocomense (Trematoda, Echinostomatidae) Trudy. Gel'mint. Lab., 21, 31-35.

Kotlan A. (1922) : Beiträge zur Kenntnis der Trematoden. Zool. Jahrb., Jena, Abt. Syst., 45, 565-576.

Ku C. T. (1938) : New trematodes from Chinese birds. Peking Nat. Hist. Bull., 13, 129-136.

Linton E. (1928) : Notes on trematode parasites of birds. Proc. U.S. Nat. Mus., 73, 1-36.

Marin R. A. (1928): Studies on schistosomiasis (S. mansoni) in Porto-Rico. Porto Rico Rev. publ. Health. Trop. Med., 3, 307-402.

Nasir P., Gonzales G., Diaz M. T. (1972) : Freshwater larval trematodes. XXX. Life cycle of Petasiger novemdecim Lutz, 1928. Proc. helminthol. Soc. Washington, 39, 162-168.

Nassi H., Pointier J.-P., Golvan Y.-J. (1979) : Bilan d'un essai de contrôle de Biomphalaria glabrata en Guadeloupe à l'aide d'un Trématode stérilisant. Ann. Parasitol. Hum. Comp., 54, 185-192.

Oshmarin P. G. (1950) : In Trematodes of Animals and Man, Echinostomatidae, Skrjabin and Bashkirova, 1956. Akad. Nauk. S.S.S.R., Moskova, Leningrad, 12, 51-930.

Pinchon R. (1963) : Faune des Antilles Françaises. Les Oiseaux. Presses Ozannes et Cie, Caen, 264 p.

Premvati G. (1968) : Echinostome trematodes from Florida birds. Proc. helminth. Soc. Washington, 35, 197-200.

Prudhoe S. (1945) : On two Echinostome Trematodes from Grebes. Ann. Mag. Nat. Hist., 12, 1-8.

Théron A. (1975): Recherches sur les rythmes d'émergence des cercaires de Guadeloupe (parasites de Biomphalaria glabrata) et des Pyrénées. Thèse de Doctorat de Spécialité. Université de Montpellier, $149 \mathrm{p}$.

Yamaguti S. (1958) : Systema Helminthum. Vol. I. Intersciences Publishers, Inc., New York, 1575 p. 